\title{
DUNFORD-PETTIS OPERATORS AND WEAK RADON-NIKODÝM SETS
}

\author{
LAWRENCE H. RIDDLE ${ }^{1}$
}

\begin{abstract}
Let $K$ be a weak*-compact convex subset of a Banach space $X$. If every Dunford-Pettis operator from $L_{1}[0,1]$ into $X^{*}$ that maps the set $\left\{\chi_{E} / \mu(E): E\right.$ measurable, $\left.\mu(E)>0\right\}$ into $K$ has a Pettis derivative, then $K$ is a weak Radon-Nikodým set. This positive answer to a question of $M$. Talagrand localizes a result of E. Saab.
\end{abstract}

In [6] Elias Saab showed that if every Dunford-Pettis operator from $L_{1}[0,1]$ into the dual of a Banach space $X$ has a Pettis derivative, then every operator from $L_{1}[0,1]$ into $X^{*}$ has a Pettis derivative so that consequently $X^{*}$ has the weak Radon-Nikodým property. The purpose of the present note is to answer a question of Talagrand by showing that this result localizes to weak ${ }^{*}$-compact convex subsets of arbitrary dual spaces.

Let $(\Omega, \Sigma, \mu)$ be a finite measure space. A function $f$ from $\Omega$ into a Banach space $X$ is said to be Pettis integrable if the scalar function $x^{*} f(\cdot)$ is integrable for each $x^{*}$ in the dual space $X^{*}$ and if for each measurable set $E$ in $\Sigma$ there is an element $x_{E}$ of $X$ that satisfies

$$
x^{*}\left(x_{E}\right)=\int_{E} x^{*} f d \mu
$$

for every $x^{*}$ in $X^{*}$. In this case we write $x_{E}=$ Pettis- $\int_{E} f d \mu$. A bounded subset $K$ of $X$ is called a weak Radon-Nikodým set [3] if for every finite measure space $(\Omega, \Sigma, \mu)$ and every bounded operator $S: L_{1}(\mu) \rightarrow X$ for which $S\left(\chi_{E}\right) \in \mu(E) K$ for every $E$ in $\Sigma$, there exists a Pettis integrable function $f: \Omega \rightarrow K$ such that $S(\psi)=$ Pettis- $\int \psi f d \mu$ for every $\psi$ in $L_{1}(\mu)$. Such a function $f$ is called a Pettis derivative of the operator $S$. The Banach space $X$ has the weak Radon-Nikodým property if its closed unit ball, $B_{X}$, is a weak Radon-Nikodým set.

For the rest of this note we shall always take $K$ to be a weak* ${ }^{*}$-compact convex subset of a dual space $X^{*}$.

THEOREM 1. Suppose $K$ is not a weak Radon-Nikodym set in $X^{*}$. Then there is a Dunford-Pettis operator $M: L_{1}[0,1] \rightarrow X$ : such that $M\left(\chi_{E}\right) \in \mu(E) K$ for all Lebesgue measurable subsets $E$ of $[0,1]$ but such that $M$ does not have a Pettis derivative.

PROOF. We may assume without loss of generality that $0 \in K$. Since $K$ fails the weak Radon-Nikodým property, then so does the weak*-compact absolutely convex

Received by the editors July 27, 1983. Presented to the Society, January 25, 1984 at the annual meeting in Louisville.

1980 Mathematics Subject Classification. Primary 46B22, 46G10.

Key words and phrases. Dunford-Pettis operators, weak Radon-Nikodým sets, Pettis integral.

${ }^{1}$ This research was supported by a Summer Fellowship from the Emory University Research Committee.

(C) 1984 American Mathematical Society $0002-9939 / 84 \$ 1.00+\$ .25$ per page 
set $K-K[\mathbf{3}]$. Let $Y$ be the Banach space of continuous functions on $K-K$ and define a bounded linear operator $T: X \rightarrow Y$ by $T x\left(x^{*}\right)=x^{*}(x)$ for every $x^{*}$ in $K-K$. Then $T^{*}\left(B_{Y^{*}}\right)=K-K$. Because $T^{*}\left(B_{Y^{*}}\right)$ is not a weak Radon-Nikodým set in $X^{*}$, the set $T\left(B_{X}\right)$ is not weakly precompact in $Y[\mathbf{3}]$. (This may be thought of as a localization of the result that a Banach space does not contain a copy of $l_{1}$ if and only if its dual space has the weak Radon-Nikodým property.)

By Rosenthal's fundamental result $[\mathbf{4}]$ on weakly precompact sets. one can find a sequence $\left(T x_{n}\right)$ in $T\left(B_{X}\right)$ that is equivalent to the usual $l_{1}$-basis $\left(e_{n}\right)$. Let $Y_{0}$ be the closed subspace of $Y$ spanned by the sequence $\left(T x_{n}\right)$ and define $W: l_{1} \rightarrow Y_{0}$ by $W\left(e_{n}\right)=T x_{n}$. Then $W$ defines an isomorphism from $l_{1}$ onto $Y_{0}$, hence its adjoint $W^{*}: Y_{0}^{*} \rightarrow l_{\infty}$ has a bounded inverse. Define $R: l_{1} \rightarrow X$ by $R\left(e_{n}\right)=x_{n}$ and let $i: Y_{0} \rightarrow Y$ denote the natural inclusion map. A moment's reflection reveals that $R^{*} T^{*}=W^{*} i^{*}$.

Now $Y_{0}^{*}$ fails the weak Radon-Nikodým property since $l_{1}$ embeds in $Y_{0}$. By Saab's global result, then, there exists a Dunford-Pettis operator from $L_{1}[0,1]$ into $Y_{0}^{*}$ that is not Pettis representable. The operator that Saab constructed in [6] has the form $Q P N$ where $N, P$ and $Q$ are defined on the spaces illustrated in the following diagram (and the other operators are to be specified shortly).

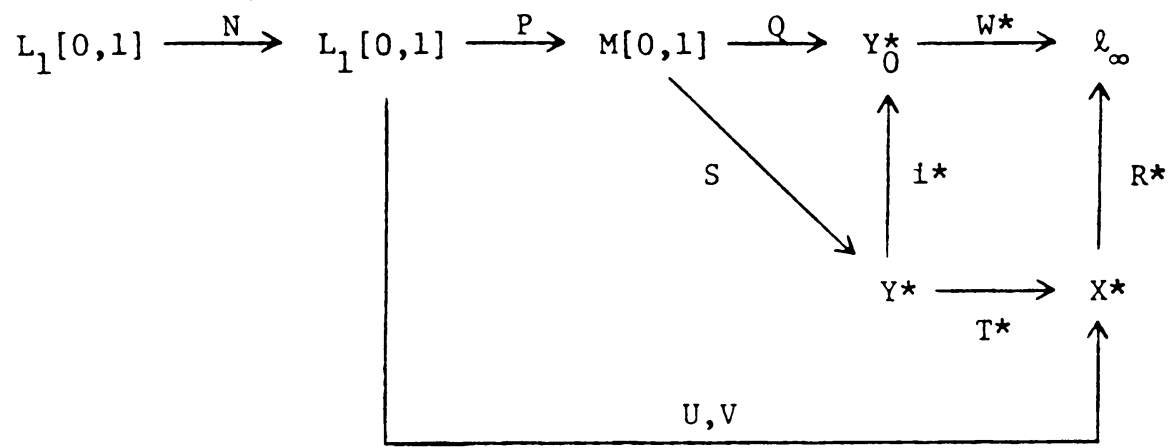

The operator $N$ is a Dunford-Pettis operator from $L_{1}[0,1]$ into $L_{1}[0,1]$ but $Q P N$ : $L_{1}[0,1] \rightarrow Y_{0}^{*}$ has no Pettis derivative. We may assume that all three operators have norm 1 . Because $M[0,1]=C[0,1]^{*}$ is an $L$-space, the operator $Q$ has a norm-preserving lifting $S$ from $M[0,1]$ to $Y^{*}$ such that $i^{*} S=Q[\mathbf{2}$, Theorem 2].

Since $T^{*} S P\left(\chi_{E} / \mu(E)\right)$ belongs to $T^{*}\left(B_{Y^{*}}\right)=K-K$, a splitting argument due to E. Saab [5] and based on a compactness argument of Lindenstrauss produces two operators $U, V: L_{1}[0,1] \rightarrow X^{*}$ such that $U\left(\chi_{E} / \mu(E)\right)$ and $V\left(\chi_{E} / \mu(E)\right)$ belong to $K$ and such that $T^{*} S P=U-V$. Bourgain has shown $[\mathbf{1}]$ that the positive part of a Dunford-Pettis operator from $L_{1}[0,1]$ into $L_{1}[0,1]$ is also a Dunford-Pettis operator. We may therefore assume that $N$ is a positive operator, for if both $Q P N^{+}$and $Q P N^{-}$are Pettis representable then so is $Q P N$. Thus

$$
N\left(\chi_{E} / \mu(E)\right) \subset\left\{f \in L_{1}[0,1]: f \geq 0,\|f\| \leq 1\right\}=P .
$$

Because 0 belongs to $K$ and because $U\left(\chi_{E} / \mu(E)\right) \in K$ for all nonnull measurable sets $E$, a convexity argument shows that $U(\mathcal{P}) \subset K$. Therefore we have $U N\left(\chi_{E} / \mu(E)\right) \in K$ and similarly $V N\left(\chi_{E} / \mu(E)\right) \in K$ for all nonnull measurable sets. Also notice that both $U N$ and $V N$ are Dunford-Pettis operators.

Now suppose both $U N$ and $V N$ have a Pettis derivative. Then so does $T^{*} S P N=$ 
$U N-V N$ and also $R^{*} T^{*} S P N$. But $R^{*} T^{*} S P N=W^{*} i^{*} S P N=W^{*} Q P N$ and $W^{*}$ has a bounded inverse, implying that $Q P N$ would have a Pettis derivative. This contradiction shows that either $M=U N$ or $M=V N$ must fail to have a Pettis derivative and finishes the proof.

The set $K$ has the weak Radon-Nikodým property if every operator taking the normalized characteristic functions into $K$ has a Pettis derivative. This is also equivalent to requiring that all operators taking the normalized characteristic functions into $K$ be Dunford-Pettis operators [5]. We now see that Theorem 1 connects these two characterizations in the following sense.

THEOREM 2. A weak*-compact convex subset $K$ of a dual space $X^{*}$ is a weak Radon-Nikodým set if and only if every Dunford-Pettis operator from $L_{1}[0,1]$ into $X^{*}$ that maps the normalized characteristic functions into $K$ has a Pettis derivative.

\section{REFERENCES}

1. J. Bourgain, Dunford-Pettis operators on $L_{1}$ and the Radon-Nikodym property, Israel J. Math. 37 (1980), 34-47.

2. K. Musial and C. Ryll-Nardzewski, Liftings of vector measures and their applications to RNP and WRNP, Vector Space Measures and Applications, II (Proceedings, Dublin 1977), Lecture Notes in Math., vol. 645, Springer-Verlag, Berlin and New York, 1978.

3. L. H. Riddle, E. Saab and J. J. Uhl, Jr., Sets with the weak Radon-Nikodým property in dual Banach spaces, Indiana Univ. Math. J. 32 (1983), 527-542.

4. H. P. Rosenthal, $A$ characterization of Banach spaces containing $l_{1}$, Proc. Nat. Acad. Sci. U.S.A. 71 (1974), 2411-2413.

5. E. Saab, Some characterizations of weak Radon-Nikodým sets, Proc. Amer. Math. Soc. 86 (1982), 307-311.

6. $363-365$.

Department of Mathematics and Computer SCIENCE, Emory University, ATLANTA, GEORGIA 30322 